\section{Fortschritte beim strukturierten Arztbrief}

Die Handhabung elektronischer Arztbriefe nähert sich langsam einem Standard, der sowohl der Sicherheit der Daten Rechnung trägt, als auch in den Abläufen wirtschaftlich ist. Vorreiter sind Ärzte im rheinischen Düren: Sie können Arztbriefe, die über die Praxis-Software erstellt sind, in ein PDF-Dokument konvertieren und dann mit Hilfe eines elektronischen Heilberufeausweises per Stapelsignatur verschlüsselt verschicken. Der Arztbrief ist Teil eines Projekts zur Entwicklung der einrichtungsübergreifenden E-Patientenakte. Die Software von Duria ermöglicht es, mehrere Dokumente auf einmal zu signieren. iss

\section{Kündigen per Einschreiben ist riskant}

Eine Kündigung per Übergabe-Einschreiben ist ein Risiko für Arbeitgeber. Holt der Arbeitnehmer die Sendung nicht ab, gilt die Kündigung als nicht zugegangen, wie das Landesarbeitsgericht (LAG) RheinlandPfalz kürzlich entschied (10 Sa 156/11). Damit gab das LAG einer Klägerin recht. Der Arbeitgeber wollte ihr mit ÜbergabeEinschreiben kündigen. Ein solches Einschreiben wird persönlich zugestellt. Weil niemand aufmachte, hinterließ der Postbote eine Benachrichtigung. Die Klägerin holte das Schreiben aber nicht ab. Daher ist es ihr auch nicht zugegangen und die Kündigung unwirksam, so das LAG. mwo

\section{Praxischef muss Überstunden verhindern oder bezahlen}

Ärzte, die Mitarbeiter sehenden Auges Überstunden machen lassen, müssen diese auch bezahlen, so das Landesarbeitsgerichts (LAG) Berlin-Brandenburg (6 Sa 1941/11). Das LAG sprach einer Angestellten $4.370 €$ für 372 Überstunden zu. Zu Beginn des Arbeitsverhältnisses hatte ihr Vorgesetzter sie angewiesen, Beginn und Ende ihrer täglichen Arbeitszeit aufzuschreiben. Das tat sie auch. Die Firma wollte die dokumentierten Überstunden aber nicht bezahlen. Das muss sie laut LAG aber. Denn der Chef habe die Überstunden geduldet und hingenommen, dass die Angestellte über ihre reguläre Arbeitszeit hinaus im Betrieb war.

\title{
Schäuble will Steuerzuschuss an Gesundheitsfonds kürzen
}

B ei der Aufstellung des Bundeshaushaltes für das Jahr 2013 plant das Bundesfinanzministerium den Steuerzuschuss an den Gesundheitsfonds von derzeit 14 Milliarden $€$ um 2 Milliarden $€$ zu kürzen. Dahinter steht offenkundig das Ziel, den Bundeshaushalt zu konsolidieren und die Nettoneuverschuldung zurückzufahren.

Angesichts sich gut entwickelnder Beitragseinnahmen der gesetzlichen Krankenversicherung - zum Jahresende 2011 hatten die Krankenkassen konsolidiert einen Überschuss von rund 4 Milliarden $€$ - würde eine Kürzung des Steuerzuschusses in der Logik des Finanzierungssystems liegen: So war im Krisenjahr 2009 der Zuschuss an den Gesundheitsfonds außerplanmäßig um rund 6 Milliarden $€$ aufgestockt, der allgemeine Beitragssatz um 0,5 Prozentpunkte gesenkt worden. Daran wollen das Bundesgesundheitsministerium und der GKV-Spitzenverband aber nur ungern erinnert werden. Beide lehnen eine Kürzung des Steuerzuschusses somit ab.

\section{Familienversicherung, Versicherten- prämien oder Polster für 2013?}

Der GKV-Spitzenverband argumentiert, die Steuermittel dienten zur Finanzierung der beitragsfreien Familienversicherung und versicherungsfremder Leistungen. Im Bundesgesundheitsministerium und bei Gesundheitspolitikern der Union ist man der Auffassung, Krankenkassen mit Überschüssen sollten nun Prämien an ihre Versicherten ausschütten. Die KBV und die KVen wollen die Überschüsse hingegen für die vertragsärztliche Versorgung mobilisieren. Dagegen kommt eine Prognose der Unternehmensberatung McKinsey zu dem Ergebnis, dass zwischen Einnahmen- und Ausgabenentwicklung eine Lücke von gut 4 Milliarden $€$ klafft, so dass Ende 2012 die Überschüsse aufgezehrt sein und spätestens 2013 ein Defizit entstehen könnte.

Helmut Laschet

\section{Arzneirabatte - geheim oder transparent?}

m Zusammenhang mit den nun angelaufenen ersten Verhandlungen über Erstattungsbeträge als Folge der frühen Nutzenbewertung für Arzneimittel mit neuen Wirkstoffen wird darüber diskutiert, ob die ausgehandelten oder von der

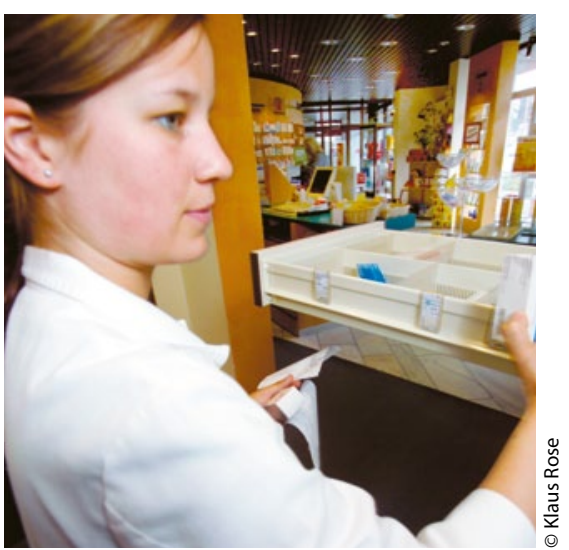

Jegliche Rabattierung von Arzneimitteln erschwert nicht nur die Ausgabe der Medikamente, sie ist auch nicht transparent.
Schiedsstelle festgesetzten Abschläge vom ursprünglichen Herstellerpreis geheim bleiben oder transparent gemacht werden müssen. Der Verband Forschender Arzneimittelhersteller (vfa) argumentiert, dass bei Offenlegung der Rabatte das internationale Preisniveau innovativer Arzneimittel ins Rutschen komme und daher die Rabatte - genauso wie bei Individualvereinbarungen zwischen Herstellern und einzelnen Kassen - vertraulich bleiben müssen. Dies sei auch gängige Praxis in anderen europäischen Ländern.

Praktikabel ist die Vertraulichkeit im Sachleistungssystem der GKV, bei der die Versicherten selbst nicht in die Bezahlung von Leistungen involviert sind. Die Rabatte nach dem AMNOG gelten aber auch für Selbstzahler der PKV und der Beihilfe. Im Moment ist offen, wie für dieses Marktsegment die Vertraulichkeit von Rabatten hergestellt werden kann.

Helmut Laschet 